\title{
Collaborative Proposal: DUSEL R\&D at the Kimballton Underground Facility (ICP-MS Confirmation, Material Assay, and Radon Reduction) \\ Final Report
}

\author{
Reporting period start date $-7 / 15 / 2007$ \\ Reporting period end date $-7 / 14 / 2010$ \\ Principle author - Dr. Henning Olling Back \\ Report issued - November 2010 \\ DOE grant number - DE-FG02-07ER41484 \\ North Carolina State University \\ Riddick Hall, Box 8202 \\ 2401 Stinson Drive \\ Raleigh, NC 27695-8202
}

\section{Executive summary:}

Experiments measuring rare events, such as neutrinoless double beta $(0 v \beta \beta)$ decay, and those searching for, or measuring very weakly interacting particles, such as low energy solar neutrino experiments or direct dark matter searches, require ever lower backgrounds; particularly those from radioactive contamination of detector materials. The underground physics community strives to identify and develop materials with radioactive contamination at permissible levels, and to remove radioactive contaminants from materials, but each such material represents a separate dedicated research and development effort. This project attempted to help these research communities by expanding the capabilities in the United States, for indentifying low levels of radioactive contamination in detector materials through gamma ray spectroscopy. Additionally the project tried to make a cross comparison between well established gamma ray spectroscopy techniques for identifying radioactive contaminations and Inductively Coupled Plasma Mass Spectroscopy, which is a relatively new method for searching for uranium and thorium in materials. The project also studied the removal of radioactive radon gas for laboratory air, which showed that an inexpensive technologically simple radon scrubber can potentially be used for homes or businesses with high radon levels even after the employment of other mitigation techniques. 


\section{Introduction}

This was a collaborative effort between Prof. Reyco Henning at the University of North Carolina (UNC), Prof. Henning O. Back at North Carolina State University (NC State), and Dick Lindstrom, formerly from NIST, as an unfunded collaborator for the gamma assay at Kimballton portion of the grant. UNC is funded through NSF and is reporting their efforts and I will be reporting on the DOE-HEP funded efforts at NC State, which have overlap with UNC's work. The proposal had three main goals:

1.) Commission and operate low-background counting systems at the Kimballton Underground Research Facility (KURF) in Virginia. Participate in the operation of the low-background counting facilities at Laboratori Nazionali del Gran Sasso in Gran Sasso, Italy.

2.) Perform a cross-comparison between Inductively Coupled Mass Spectroscopy (ICPMS) and the gamma-assay technique.

3.) Systematically study techniques to reduce Radon in laboratory in air.

After the grant was awarded Prof. Henning and Prof. Back decided that UNC would pursue task 2, while NC State would focus on task 3. Both PIs participate in Task 1. Additionally, by taking advantage of facilities at NC State, Prof. Back tried to identify materials for task 2.

Task 1 was a large success, with a publication submitted to NIM A. KURF is now accepted in the neutrino and dark matter communities as a reliable material assay facility. This included commissioning detectors and the assay techniques, as well as significant facility upgrades in safety and air quality provided by NC State. Also, the NRL01 detector in Gran Sasso was made operational, and materials were counted for the Majorana experiment. Because of the efforts of this project, the NRL01 detector is now in continuous use at Gran Sasso, after having been largely abandoned.

Task 2 is an effort still being pursued by the UNC group, however at NC State we took advantage of the Instrumental Neutron Activation Analysis (INAA) facilities in the NC State Nuclear Engineering Department to attempt to identify materials for this cross comparison. As a result NC State was able to make a competitive determination of uranium and thorium content in a plastic that had been assayed via another INAA method at the University of Alabama. A publication of these results is in preparation.

Troubles with air quality at KURF, and struggles with detector development, significantly delayed the radon reduction studies of task 3 . In the end we did produce a radon scrubbing system for reducing the radon levels in laboratory air. The decrease in radon was not what we were striving for, but the levels would be useful for residences or businesses that are struggling to reduce the radon in their air through other mitigation techniques.

\section{1) Commission and operate low-background counting systems at the Kimballton Underground Research Facility (KURF)}

UNC has taken the lead in the counting systems at KURF in this collaborative effort; however, NC State has also had a significant role with this work.

\section{Laboratory:}

The laboratory space available at the beginning of the project was a 24 ' x 8' ISO container on a concrete pad (Figure 1). The ISO container had been exposed to the high levels of 
mine dust for several years, and it was immediately clear that the dust levels needed to be mitigated. During the summer and fall of 2007 a metal building (100' x 36' x 20') was erected on the concrete pad, with man-power help from NC State, to reduce the dust levels, and to define the KURF laboratory space (Figure 2). Since the construction of the laboratory building several other collaborations have moved experiments into the KURF (Figure 3).

Although the building significantly reduced the dust levels, particularly in the ISO container laboratory, it was still clear that steps were needed to decrease the influx of dust and to filter the air inside KURF. Virginia Tech had installed an air filter that uses paper filters. However, although this method is effective, the filters need to be changed very often; every couple days is best. Therefore, at NC State we developed an air filter based on water mist for the radon scrubbing unit. This method was so successful that Virginia Tech scaled up the design and built a larger scale version for the entire building. The water mist air filter is detailed below in the section entitled "Systematically study techniques to reduce Radon in laboratory in air".

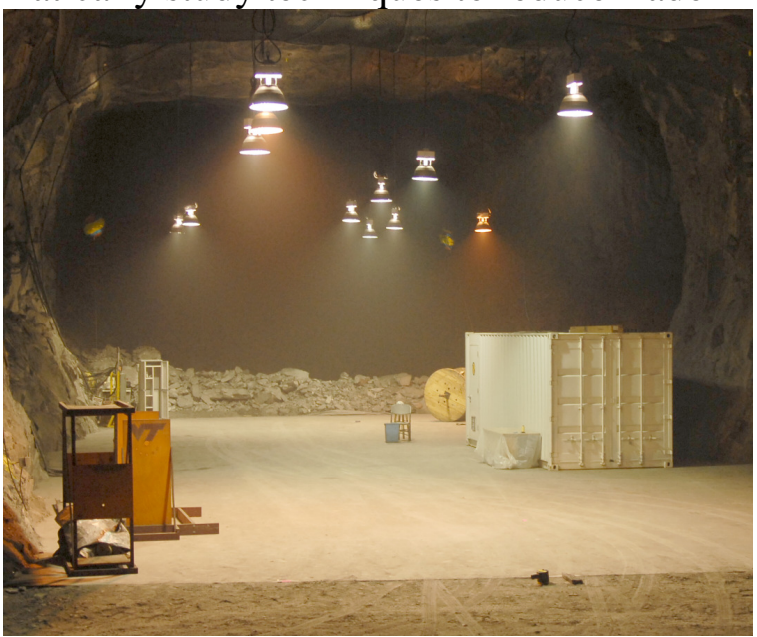

Figure 1: KURF lab in 2007 with ISO container and concrete pad.

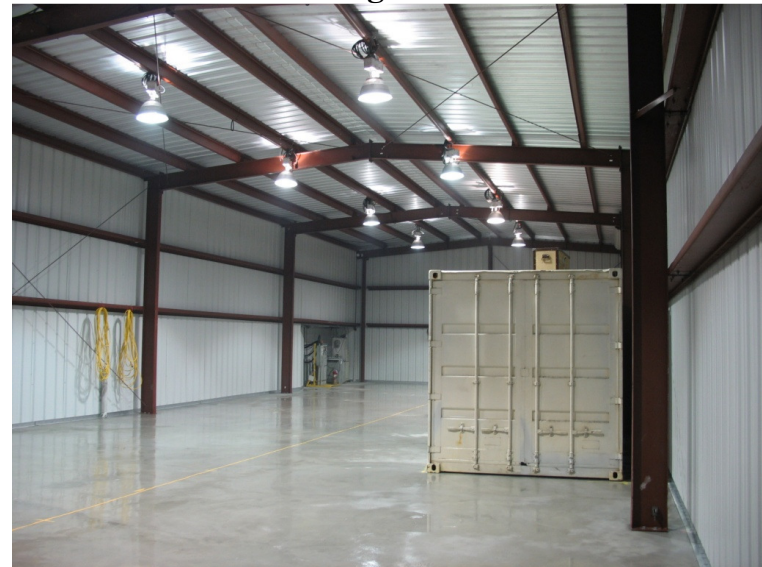

Figure 2: KURF in 2008 with metal building constructed to define laboratory and reduce dust.

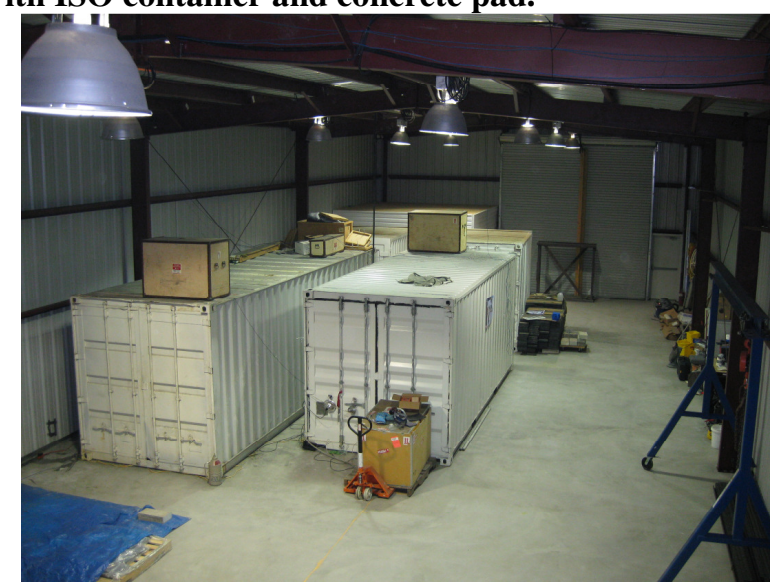

Figure 3: KURF in 2009; expanded to with two other experiments.

NC State also designed, built and installed an oxygen safety monitor system for the NRL ISO container laboratory. The two germanium detectors in the laboratory use liquid nitrogen for cooling, and there is an oxygen deficiency hazard, especially when the detectors are filling. In order to comply with MSHA regulations and for common sense safety we designed and installed an oxygen concentration monitoring system with alarms. This system turns on a fan into the 
detector trailer when the oxygen drops to a low, but safe level, and sounds an alarm ordering people to evacuate if the oxygen level falls below an unsafe level. This system was duplicated by the MALBEK collaboration for the ISO containers for their experiment, which is located in KURF.

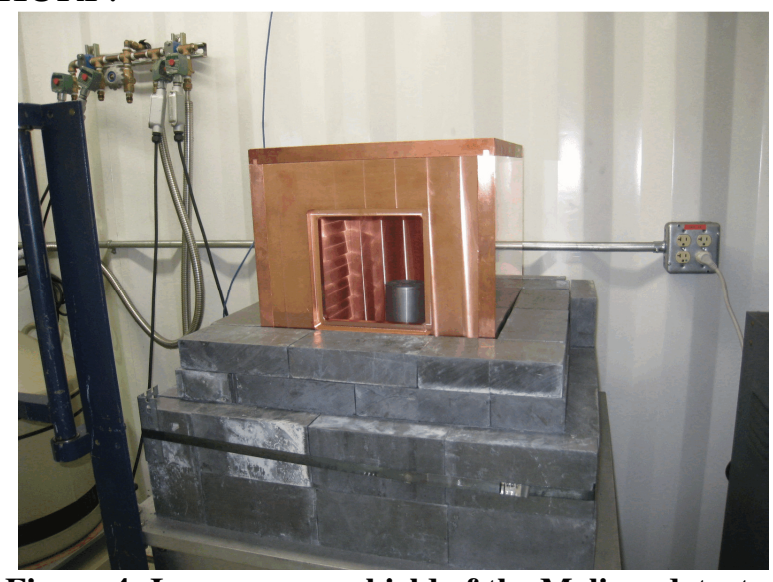

Figure 4: Inner copper shield of the Melissa detector

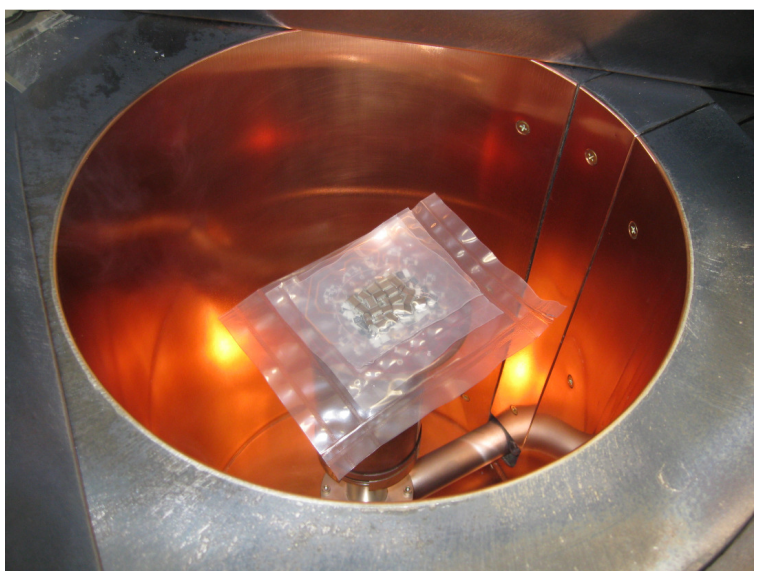

Figure 5: Interior of the VT-1 detector

\section{Detectors:}

Melissa is a commercial low-background detector with an offset cryostat and an outer can made from selected low-background aluminum. It was manufactured by Canberra under model number GC5018 with serial number 4943314. It has a lead shield that separates the detector from the preamp. It was bought new in 1994 and repaired in 1999. Its efficiency is $52.2 \%$ relative to NaI, and it is housed in a 6" shield of Doe Run grade virgin lead bricks. It was upgraded with a copper inner shield (Figure 4) and a nitrogen purge in the detector volume to reduce radon daughter background. The detector has been running since the summer of 2008 and has counted several samples by the UNC group.

An issue had arisen with Dr. Kroeger's departure from the NRL with regard to the possession of Melissa. Melissa is owned by the Air Force Technical Applications Center (AFTAC) but was part of a joint NIST and NRL project, which was terminated when Dr. Kroeger left the NRL. AFTAC would like to reclaim Melissa, but Dr. Lindstrom has been able to negotiate that Melissa stay at KURF for an indefinite period of time. Melissa stayed at KURF for the duration of this grant, and continues to be used by the UNC; who negotiated a contract extension with NSF to continue material assay at KURF.

An NC State undergraduate student, Daryl Metzler, spent the 2008 summer learning data analysis techniques with ROOT, and prepared for counting an irradiated plastic sample. The University of Washington sent us a sample of parylene plastic, which is used as a dielectric in some coaxial cables. We then had the Nuclear Engineering Department perform Instrumental Neutron Activation Analysis (INAA) on the plastic. Our curiosity was to see if the KURF detectors would be sensitive enough to see the characteristic gammas from the irradiation of Uranium-238 and Thorium-232 after the radioactivity in the plastic has dropped to levels were it was releasable to us without a license. In INAA the U and Th absorb a neutron to create shorter lived isotope, which decay in to short lived daughters that emit gammas. For example:

1) ${ }^{232} \mathrm{Th}+\mathrm{n} \rightarrow{ }^{233} \mathrm{Th}\left(\mathrm{t}_{1 / 2}=22 \mathrm{~m}\right)$

2) ${ }^{233} \mathrm{Th} \rightarrow \mathrm{e}^{-}+{ }^{233} \mathrm{~Pa}\left(\mathrm{t}_{1 / 2}=27 \mathrm{~d}\right)$

3) ${ }^{233} \mathrm{~Pa} \rightarrow \mathrm{e}^{-}+{ }^{233} \mathrm{U}\left(\mathrm{t}_{1 / 2}=1.6 \mathrm{e} 5 \mathrm{y}\right)+\gamma(312 \mathrm{keV})$ 
It's the $312 \mathrm{keV}$ gammas we searched for.

Unfortunately the time it took to get the sample released by the Nuclear Engineering department was 28 days after the irradiation; therefore the ${ }^{239} \mathrm{~Np}$ from the Uranium had gone through more than 10 half-lives, and had effectively decayed away. The ${ }^{233} \mathrm{~Pa}$ had decayed by one half-life, which was apparently enough to not be able to detect any decays above background. The NC State Nuclear Engineering NAA resulted in an upper limit of about 50 ppm for thorium-232, and therefore we don't actually know what the expected signal should be.

VT-1 (Figure 5) is an Ortec GEM series commercial low-background detector in a J-type cryostat with $35 \%$ relative efficiency. It is mounted in a 4 " commercial lead shield with an inner copper shield. The detector was repaired by Kim Lister at Argonne National Lab, and was returned to Virginia Tech in April 2008. During the 2008 summer NC State moved this detector and the 2.5 ton shield and installed it in the KURF laboratory.

The Melissa and VT-1 detectors assayed materials until the end of this grant period, and the UNC group is continuing material assay with the detector. A publication about the KURF laboratory and the material assay efforts there has been submitted to Nuclear Instruments and Methods A. The reviewer's comments have been returned and are being addressed; we expect to have the revised paper resubmitted soon.

\section{2) Participate in the operation of the low-background counting facilities at Laboratori Nazionali del Gran Sasso in Gran Sasso, Italy.}

The NRL-1/GEMPI-2 detector was developed by the Max Planck Institute für Kernphysik (MPIK) under a cooperative agreement with the Naval Research Laboratory (NRL). The detector and shield were assembled to operate at the Laboratori Nazionali del Gran Sasso (LNGS), Italy in 2005, where it is currently operated by Dr. Matthias Laubenstein. The NRL-1 detector is considered to be the best low-background HPGe detector in the world. As part of this grant we have a cooperative agreement that provides us with at least 3 months per year of time on this detector.

During the first 6 months of the project period Matthias Laubenstein commissioned the detector and began assay operations. For the Majorana Neutrinoless Double Beta Decay experiment we counted approximately $24 \mathrm{Kg}$ of copper that will be used as the precursor for their electroformed copper. This copper was cleaned and prepared at Pacific Northwest National Laboratory (PNNL) and then shipped to Gran Sasso; however upon arrival it was discovered that the clean packaging had failed and the copper had been exposed to the environment. This exposure had the potential for contaminating the copper with uranium and thorium from dust, and therefore a rigorous cleaning process was developed by PNNL and supplied to Dr. Laubenstein at Gran Sasso. After the lengthy cleaning processes, counting of the copper began in February 2009, and continued on and off through August 2009. Results of the counting were provided in October 2009, 6 months after counting began. During that time the counting was stopped for various reasons, the main reason being the earthquake that struck central Italy. The earthquake in L'Aquila Italy on Monday 4/6/2009 was very close to the Gran Sasso laboratory. The underground and above ground laboratories suffered no damage, but many of the employees live in areas that were damaged by the earthquake, and therefore there were significant delays in operations at the laboratory. Despite the delays the total counting time was greater than 125 days, more than 4 months. 
The results from Gran Sasso were:

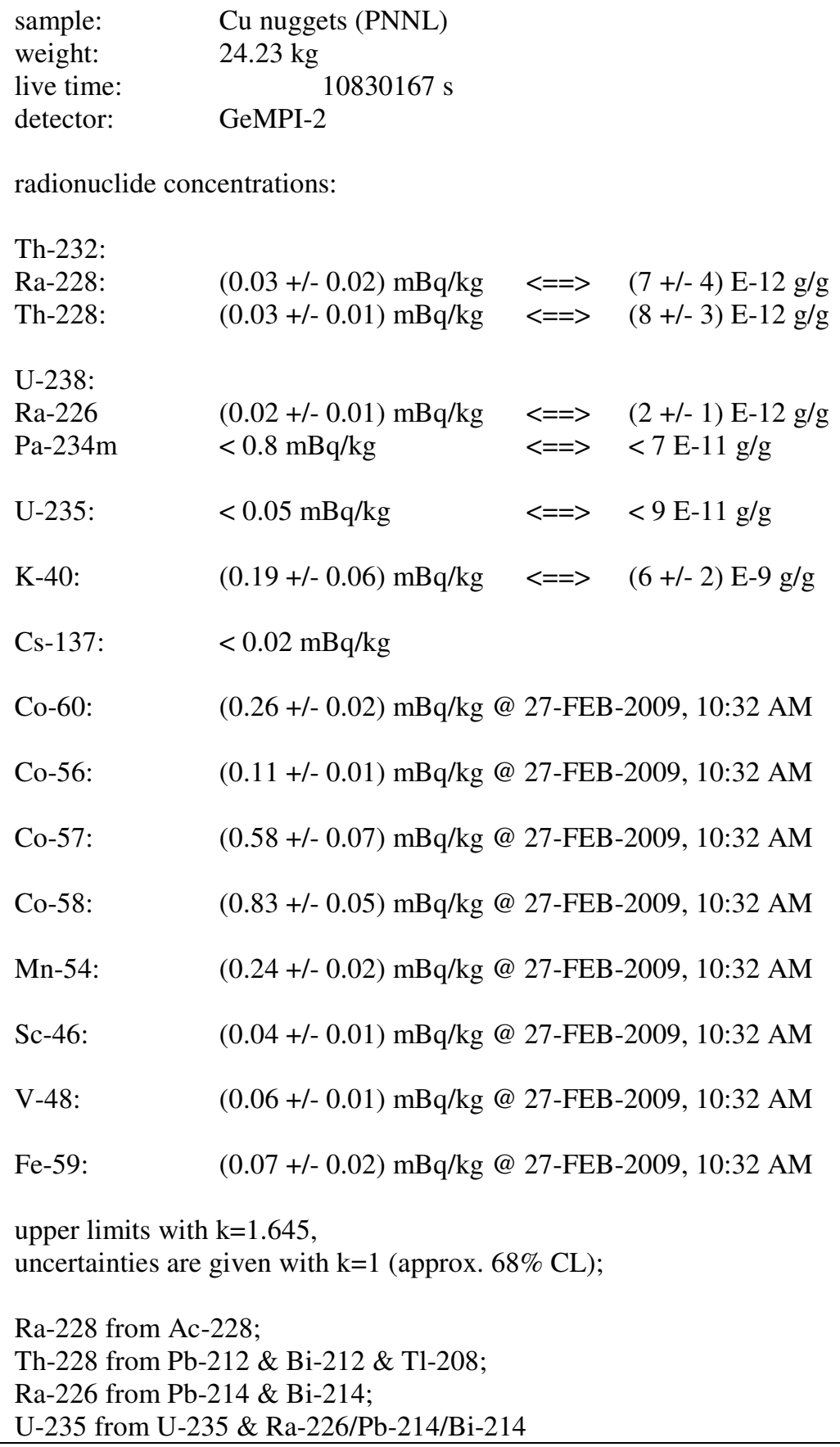

The result from counting the Majorana copper was very similar to the counting of electrolytic copper in a similar detector several years prior. Reference (i) details the measurement, but for comparison they measured ${ }^{226} \mathrm{Ra}$ to be $<0.016 \mathrm{mBq} / \mathrm{Kg}$ and ${ }^{228} \mathrm{Ra}$ to be $<0.018 \mathrm{mBq} / \mathrm{Kg}$, which are part of the ${ }^{238} \mathrm{U}$ and ${ }^{232} \mathrm{Th}$ chains respectfully. This shows that the $\mathrm{U}$ and Th levels are at least as good as the electrolytic copper used in the GERDA experiment. The primary reason for this counting was to search for any unknown gamma backgrounds that may 
exist in the copper for Majorana. There were some short lived gamma emitters, but they are produced from cosmic rays in the copper, from flying to Italy, and surface exposure.

No other samples we counted in NRL-1 during the project period. Flying materials to Italy can cause the ingrowth of cosmic ray produced radio isotopes, which the experiments we contacted were afraid of. Also, counting time on NRL-1 is reserved for materials were the radio purity requirement is very stringent (i.e. the Majorana copper), which limits what can be counted. If the requirements are not very stringent then it can be assayed in other detector, for example the detectors in KURF. Because of these requirements we were not able to identify a material from the DUSEL collaborators to be assayed in the NRL-1 detector.

\section{3) Perform a cross-comparison between Inductively Coupled Mass Spectroscopy (ICP-MS) and the gamma-assay technique}

Although UNC is the lead institution for this effort, at NC State we attempted to identify plastics that could potentially be used for this effort, and be useful to the Majorana experiment. We decided to begin by assaying the plastics through Instrumental Neutron Activation Analysis (INAA) to determine the ${ }^{232} \mathrm{Th}$ and ${ }^{238} \mathrm{U}$ levels. For the first plastic, Parylene, we did not reach sensitive enough levels to determine the actual ${ }^{232} \mathrm{Th}$ and ${ }^{238} \mathrm{U}$ content to be useful for ICP-MS cross-comparison, and the second plastic, TE-6472 Teflon, had such low levels of ${ }^{232} \mathrm{Th}$ and ${ }^{238} \mathrm{U}$ the INAA could not determine the actual concentration.

INAA is a trace element analysis processes where a sample is exposed to a high neutron flux, primarily from a nuclear reactor, and the element being searched for captures a neutron and becomes a short lived gamma emitting radioactive isotope. The sample is then measured with a gamma detector and signature gamma lines are identified. By determining the short-lived radioactive isotope decay rate, and understanding detector efficiency, neutron flux through the sample, and neutron capture cross sections, the concentration of the element in question can be calculated. This method relies on either calculating the neutron flux at the irradiation position, or measuring it with a neutron flux monitor. It also relies on understanding the neutron capture cross sections, the neutron moderation in the media being analyzed, and on the counting geometry.

At NC State the INAA method eliminates the need to precisely understand the neutron flux during the irradiation by irradiating a known standard of the element. This standard is not used to calculate the neutron flux, but rather to determine the neutron capture rate on the element. This method therefore illuminates the need for knowing the neutron flux precisely and does not rely on a good understanding of the neutron capture cross section. Additionally, by creating a standard that is in the same geometry as the sample, the gamma detection efficiencies also cancel during the gamma counting

The PULSTAR reactor in the Nuclear Engineering Department at NC State is a 1MW pool-type reactor used for research and education at NC State. INAA is a service that is provided for a fee at the PULSTAR reactor through the Nuclear Services (http://www.ne.ncsu.edu/NRP/naa.html). Samples are prepared and placed in plastic activation vials, which are then heat sealed and packed into a plastic bottle 5 at a time. These bottles are loaded into aluminum stingers and are placed next to the reactor core for irradiation.

INAA at NC State has been used to determine ${ }^{238} \mathrm{U}$ and ${ }^{232} \mathrm{Th}$ concentration in the past; however, not at the ultra-low levels that Majorana requires $(\mu \mathrm{Bq} / \mathrm{Kg})$. In July of 2008 an initial assessment of the INAA capabilities at NC State for U and Th was performed on samples of Parylene plastic. The limits obtained from the Parylene were not as low as what Majorana or the 
ICP-MS cross comparison require. For the TE-6472 Teflon we attempted to reduce the upper limits by increasing the sample mass, increase the irradiation time, and decrease the time after irradiation to begin counting (for $U$ determination).

The upper limit for ${ }^{238} \mathrm{U}$ contamination was determined to be $2.77 \mathrm{ppt}$ and the upper limit for ${ }^{232}$ Th contamination was $0.809 \mathrm{ppt}$. These results are consistent with limits obtained by INAA performed by colleagues on the EXO experiment (ii), and are pushing the INAA limits for ${ }^{232} \mathrm{Th}$ and ${ }^{238} \mathrm{U}$ determination. Since this is the first time this method has been used to determine such low limits a publication is in preparation for the TE-6472 Teflon INAA.

\section{4) Systematically study techniques to reduce Radon in laboratory in air.}

The goal of the radon scrubbing portion of the grant has always been to produce a radon reduced laboratory space at Kimballton. We planned to perform some tests for the radon scrubber while working toward that goal. But we hit several obstacles.

Sean Finch, an undergraduate from NC State, was an REU student at the Triangle Universities Nuclear Laboratory (TUNL) during the 2008 summer, and spent the summer working on the radon reduction project. Being an NC State student Sean continued to work on this project throughout the school year as well as the 2009 summer. We created the test columns of varying size to study charcoal column shape versus radon reduction efficiency; however the radon levels in the TUNL laboratory are on the order of $1 \mathrm{pCi} / \mathrm{L}$ and fluctuate by $50 \%$ or more. Although we tried various controls, the fluctuations continued. In addition to the fluctuations, the radon level is very low and if we expect radon reduction efficiencies on the order of 100 to 1,000 times, or even 10 times, then the radon level exiting the absorption column will be lower than the $0.1 \mathrm{pCi} / \mathrm{L}$ lower limit of the commercial radon detector we have. We determined that this study can only really work with a radon source, but these sources are prohibitively expensive. The development of a low level radon detector was plagued with noisy electronics issues and was man power intensive. Although progress was made, we were not successful in reproducing one.

When the proposal was written the only radon scrubbers thought to be in existence did not produce the radon levels as they hoped for. However, it turns out that there was one that was producing radon reduced air in large volumes, but had not published its results. The NEMO experiment in Modane France is producing air that has radon 100 times lower than the ambient air at a rate of $150 \mathrm{~m}^{3} / \mathrm{hr}$ (iii). The techniques are straight forward, and we wanted to recreate this radon scrubber; however, the system requires very cold air and pressure vessels. The only air chiller that we found available is very expensive and required very clean air for its input. We also learned that there are significant MSHA regulations for pressure vessels in mines. The high dust levels in KURF will need to be mitigated before we build a radon scrubber with an air chiller, and because of the overhead needed for the pressure vessel regulations we attempted to make a radon scrubber at ambient pressure and temperature.

The high dust levels need to be mitigated before we can move toward a radon scrubber with chilled air. Steps have been made to reduce the dust levels, and for the most part the dust levels have decreased. However, for the radon scrubber the dust needed to be reduced further, and at NC State we developed an air filter based on water mist for this purpose. Not only would an air chiller need very clean air, but the regenerative air pump also needs the air to be cleaner. The principle used for the system is to pass the air through a volume of water mist, and trap the dust on the water droplets. This method is used in large industrial units, but they are much larger 
than we need, and therefore we built a smaller scaled version. Figure 6 shows and schematic of the system. Air is passed through water mist inside of PVC tubes, and is collected in the water tank. A pneumatic pump recirculates the water through the system. The closed loop water system is required due to the lack of clean water at KURF. A pneumatic pump was originally chosen for potential safety concerns, but the air compressor cycled more than the manufacture's

recommendation, and therefore the filter was upgraded with an electric pump. Figure 7 shows a picture of the completed filter installed at KURF. The output air is noticeably cleaner, but a quantitative number is not known. Due to the success of this principle the Virginia Tech group built a larger version to supply cleaner air to the whole building.

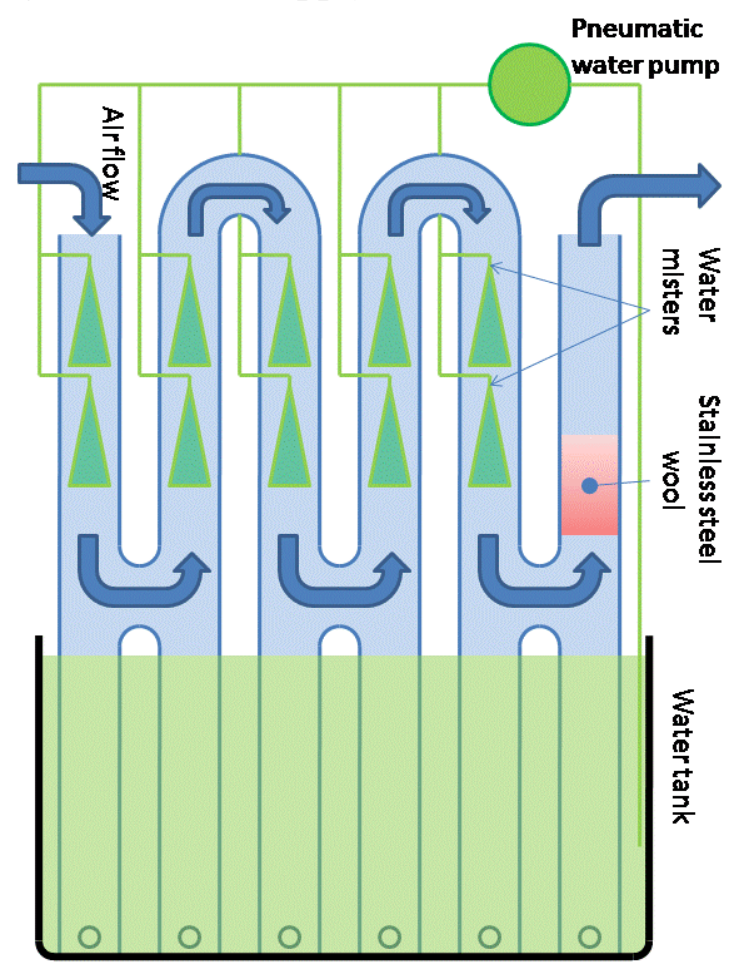

Figure 6: Water mist air filter schematic. Air flows in the direction of the blue arrows inside of PVC tubes, and water is misted inside

these tubes. The water is collected in the water tank, and recirculated through the system. The stainless steel wood traps excess water mist.

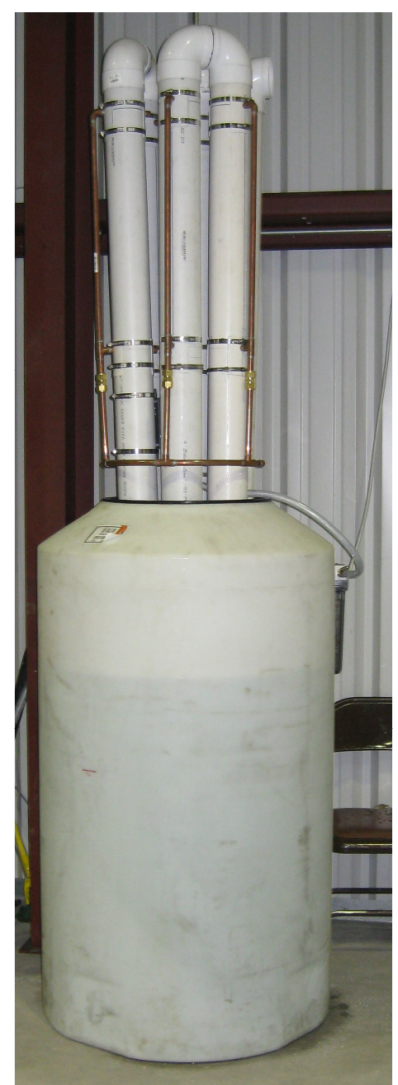

Figure 7: Water mist air filter installed at KURF

The radon scrubber was designed using commercially available low pressure charcoal columns (Figure 8). These charcoal columns have a volume of 55 gallons, and hold about $200 \mathrm{lbs}$ of charcoal each. The final scrubber consists of 5 of these columns connected in series, which gives us $1000 \mathrm{lbs}$ of charcoal to scrub the radon from the air. A regenerative air blower is used to move the air through the system, and a dehumidifier removes moisture from the air. Moisture in the air competes with the radon trapping; therefore we must dry the air before entering the charcoal columns. Figure 9 shows a schematic of the completed radon scrubbing system. 


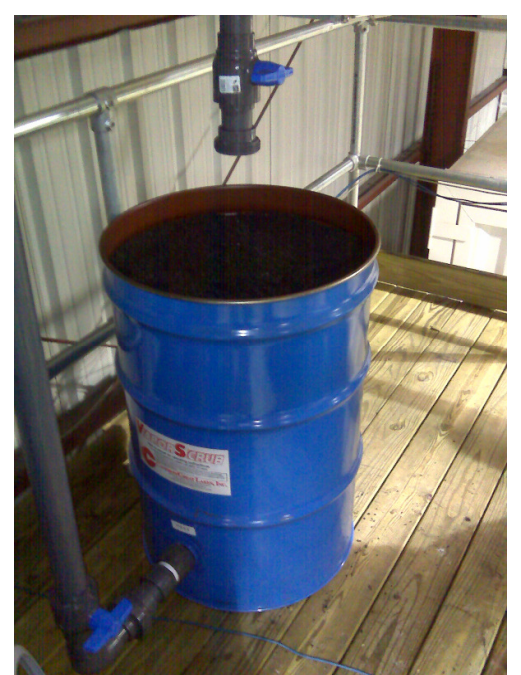

Figure 8: 200 lbs charcoal column from Cameron Carbon Inc.

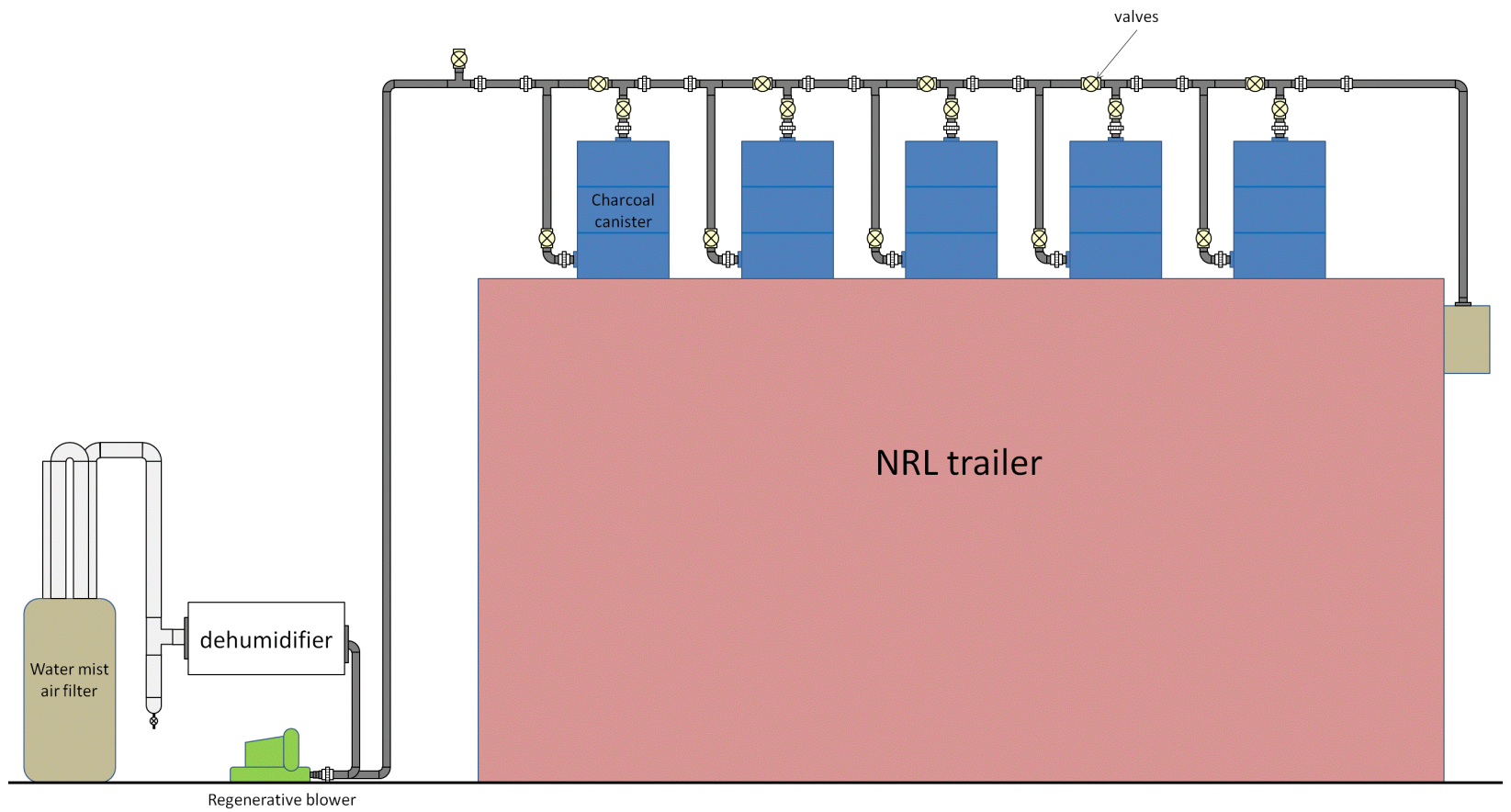

Figure 9: Schematic of the KURF radon scrubber used to reduce radon in the air for the NRL ISO container laboratory.

The radon scrubber was completed in June 2010, and the initial performance was measured. The system was first operated in bypass mode, where the air is not allowed to flow through the charcoal, and in this mode a 24 hour background run was taken as a base line. Data was then taken for more than 10 days, and immediately following this the scrubber was put back into bypass mode and background radon data was taken again. The results are shown in Figure 10 , and they show promise. It appears that radon at the outlet of the scrubber was reduced more than $30 \%$, and in fact, the post run background seems to show that the radon was reduced in the entire building, not only at the output of the scrubber. Unfortunately the project period ended before it was possible to do a longer study to confirm the performance. However, this shows that it is possible to reduce the radon in air with charcoal, at room temperature and at ambient pressure. Higher pressure and colder air will improve the efficiency, but a unit without the 
overhead of high pressure vessels, and air chillers, can find use at DUSEL and potentially in American homes where the radon is above the EPAs recommendations, or in work places where the radon levels are high.

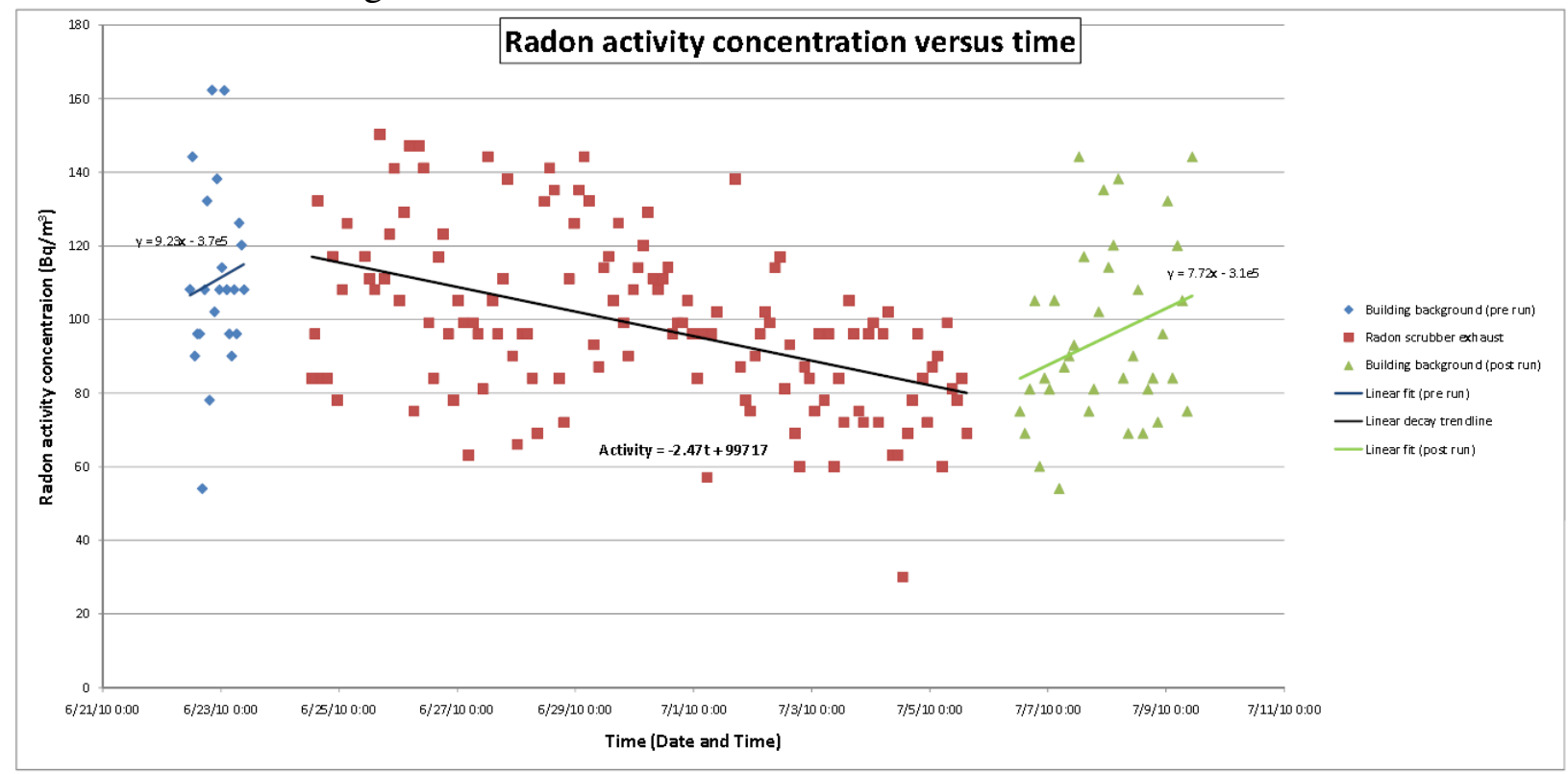

Figure 10: Radon scrubber performance data.

\section{Education and Outreach:} project.

During the 3 year grant period we were fortunate to have several students work on this

Ann Talley was a senior who worked with the project for part of the summer in 2007, and after she graduated in May 2008 she continued with the project through the summer until she took a position at the Babcock and Wilcox Company in Lynchburg, VA. Her experience with this project was a significant factor in getting that position. Ann worked on calculating radon concentration limit estimation and creating an oxygen monitor safety system for the KURF laboratory. Ann also participated directly with the Nuclear Engineering department to perform the INAA of the Parylene plastic.

Daryl Metzler was a junior that worked on gamma counting a plastic sample that had been irradiated at the NC State nuclear reactor for Neutron Activation Analysis purposes. He spent half of the summer learning Root and led the counting at KURF with the Melissa detector, which unfortunately did not produce a result for the determination of Uranium and Thorium concentrations in the irradiated plastic.

Sean Finch was an REU student at the Triangle Universities Nuclear Laboratory during the 2008 summer and was funded by the laboratory. He worked on designing and building the charcoal radon absorption columns, and learning to use a commercial radon detector we bought. He determined that the ambient radon levels would be difficult to use in the prototype charcoal column studies. Sean continued to work on the project throughout the school year, and joined us for part the 2009 summer. He will be graduated with a BS in physics in May 2009, and is now a PhD student at Duke University.

Alex Pronschinske was an NC State graduate student that worked part-time on producing a radon detector using a PIN diode during the summer of 2008. Alex's true passion was for Condensed Matter physics, which he pursued after the summer. 
Dr. Lindstrom presented efforts at KURF at the 5th International Conference on Radionuclide Metrology: Low-Level Radioactivity Measurement Techniques in Braunschweig Germany in September 2008.

Dr. Back was consulted by the DUSEL designers for including radon reduction apparatus to the cleanrooms for the Majorana demonstrator laboratory planned for the 4850' level of the Sanford Lab. He also gave a seminar about KURF at the University of New Hampshire, and presented colloquia that included KURF as a topic.

${ }^{\mathrm{i}}$ M. Laubenstein, M. Hult, J. Gasparro, D. Arnold, S. Neumaier, G. Heusser, M . K.ohler, P. Povinec, J.-L. Reyss, M. Schwaiger, P. Theodorsson, 2004. Underground measurements of radioactivity. Appl. Radiat. Isotopes $61,167-172$

${ }^{\text {ii }}$ D. S. Leonard, et Al. 2008. Systematic study of trace radioactive., Nuclear Instruments and Method A 591, pp. 490-509

iii Nachab, A. 2007. Radon reduction and radon monitoring in the NEMO experiment. In Topical Workshop on Low Radioactivity Techniques. Vol. 897. Aussois, France, 1-4 October 2006, Melville, N.Y.: American Institute of Physics. pp. 35-38 